LETTER TO THE EDITOR

\title{
Concurrent HIV and Zika Virus Infection, A Problem to be Address in HIV Medicine
}

\author{
Beuy Joob $^{1^{*}}$ and Viroj Wiwanitkit ${ }^{2}$ \\ ${ }^{1}$ Sanitation 1 Medical Academic Center, Bangkok, Thailand \\ ${ }^{2}$ Visiting Professor, Hainan Medical University, China
}

*Corresponding author: Beuy Joob, Sanitation 1 Medical Academic Center, Bangkok, Thailand, E-mail: beuyjoob@hotmail.com

Dear Editor, the HIV infection is a worldwide distributed disease at present. The infection is the most common immunodeficiency disorder and is still the global public health threat. This disease is still non-curable and the continuous research aiming at the successful management of the disease is required. Mainly, HIV is transmitted by sexually contact, blood and blood contact, transplacental from mother to infant or organ receipt. The concurrence between HIV and other medical disorder is an interesting issue in HIV medicine. Of several medical disorders, the new emerging infection should be mentioned. At present, Zika virus infection is the new emerging infection that can be transmitted as vector borne disease and also transmittable via sexual contact.

The concurrences between HIV infection and Zika virus infection are already reported [1-3]. The first case report was from Brazil [2]. There is no significant important clinical observation on the concurrence [2]. The problem in the HIV pregnant woman is also already reported [3]. João, et al. mentioned that the concurrence "led to multiple fetal malformations and fetal demise [3]". Nevertheless, due to the lack of accumulated data, it is needed to have a long - term monitoring on this problem. Wiwanitkit noted that "further accumulated data on this topic are needed. Since the Zika virus is presently proposed for the possibility of sexual transmission, the concern on the concurrence becomes the great topic [1]".

Concurrence between HIV infection and Zika virus infection might cause a new problem. Zika virus is associated with fetal anomalies, such as microcephaly, however, it is not clear if there is a concurrence of HIV, there will be any impact on this. Due to the lack of complete data at present, more collective data from researches are requires to justify the association between ZIKV and HIV infections.

\section{Conflict of Interest}

None.

\section{References}

1. Wiwanitkit V (2016) Concurrent Zika virus and HIV infection: A new concern on the new concurrence. Ann Trop Med Public Health 9: 355.

2. Calvet GA, Filippis AM, Mendonça MC, Sequeira PC, Siqueira AM, et al. (2016) First detection of autochthonous Zika virus transmission in a HIV-infected patient in Rio de Janeiro, Brazil. J Clin Virol 74: 1-3.

3. João EC, Gouvea MI, Teixeira ML, Mendes-Silva W, Esteves JS, et al. (2017) Zika virus infection associated with congenital birth defects in a HIV-infected pregnant woman. Pediatr Infect Dis J 36: 500-501. 